\title{
Bioanalyzer chips can be used interchangeably for many analyses of DNA or RNA
}

\author{
Jessica Davies, Tom Denyer, and James Hadfield \\ Genomics Core Facility, Cancer Research UK Cambridge Institute, Li Ka \\ Shing Centre, University of Cambridge, Robinson Way, Cambridge, UK
}

BioTechniques 60:197-199 (April 2016) doi 10.2144/000114403

Keywords: RNA; NGS; Bioanalyzer; QC; quality control

Supplementary material for this article is available at www.BioTechniques.com/article/114403

The Agilent 2100 Bioanalyzer enables small-scale gel electrophoretic separation of nucleic acids on a microfluidic chip; however, a shortage of chips and an excess of reagents are common issues. Here we explore the compatibility of two commonly used Bioanalyzer reagents with three Bioanalyzer chip types. Microfluidic electrophoretic separation of DNA and RNA using DNA High Sensitivity and RNA 6000 Nano reagents, respectively, was successfully performed on multiple chip types following the assay-specific protocols. For RNA quality and next-generation sequencing (NGS) library length estimation, the Bioanalyzer chips we tested can be used interchangeably. These findings will be valuable for any laboratory using the Agilent Bioanalyzer in a shared facility.

The Agilent 2100 Bioanalyzer (Agilent Technologies, Santa Clara, CA) performs microfluidic electrophoretic separation on microfabricated chips (1). In comparison to slab gel electrophoresis, the Bioanalyzer provides many advantages: separation is quick; a minimal sample volume $(1 \mu \mathrm{l})$ is required; user exposure to hazardous materials is minimal; and the assessment of sample quantity and quality is not dependent on the user's interpretation.

Results of nucleic acid sample separation are displayed on an electropherogram and a gel-like image, both generated by the Bioanalyzer 2100 Expert Software (1). These outputs provide a visualization of sample quality and quantity; for RNA, integrity is additionally assessed by a software algorithm that produces an RNA integrity number (RIN) (Agilent Application Note. Publication Number 5989-1165EN. 2004) (2). DNA samples, such as PCR products, restriction digests, and plasmid digests, can be assessed with kits covering a vast range of product lengths. Moreover, the additional highsensitivity reagents are particularly useful for library assessment prior to next-generation sequencing (NGS).

A common problem in laboratories using the Bioanalyzer is that of chip shortage with excess reagents, partic- ularly in laboratory service environments that experience both high usage and fluctuations in the demand for different kit types. Therefore, we investigated whether reagents can be used interchangeably with different Agilent Bioanalyzer chips, which would be helpful to many researchers. Anecdotal reports of using the wrong chip type have been noted previously (http://seqanswers. com/forums/showthread.php?t=41878). Others have demonstrated the ability to re-use chips multiple times without detrimentally affecting results $(3,4)$. Thus, we explored the compatibility of Agilent Technologies' RNA 6000 Nano and DNA HS Bioanalyzer reagents with three chip types, following the assayspecific protocols and using the assayspecific software.

The RIN and concentration of four RNA samples measured in triplicate (R1, R2, R3, and R4) were assessed, using the RNA 6000 Nano reagents and protocol, on RNA 6000 Nano, DNA HS, and DNA 1000 chips. Importantly, the sticker displaying the chip layout was disregarded, and the loading pattern indicated in the assay-specific protocol was used. All chips were run on the Agilent 2100 Bioanalyzer using the Eukaryotic Total RNA Nano Assay software protocol. The concentration and RIN of each RNA sample were highly comparable between chips (interchip concentration: $P=0.96$; RIN: $P=$ 0.13 by ANOVA) (Table 1 and Figure 1). Intra- and inter-chip variability for RNA RIN and concentration were very similar. RIN and RNA concentration were both well within the normal variability expected of samples used for RNA-Seq experiments.

The length distribution and concentration of four DNA samples (RNA-Seq libraries; D1, D2, D3, and D4) were assessed using the DNA HS reagents and protocol on RNA 6000 Nano, DNA HS, and DNA 1000 chips; again, the sticker displaying the chip layout was disregarded. All chips were run on the

\section{METHOD SUMMARY}

RNA and DNA samples were run in triplicate using RNA 6000 Nano and DNA High Sensitivity (HS) reagents, respectively, on RNA 6000 Nano, DNA HS, and DNA 1000 chips. The quality and concentration of RNA samples and the concentration and length distribution of DNA samples were determined. We demonstrate that any of the Bioanalyzer chips tested can be used interchangeably with defined Bioanalyzer reagents for qualitative analysis. The chips can also be reasonably quantitative, provided the protocol and software method for the corresponding assay reagent kit are followed correctly. 

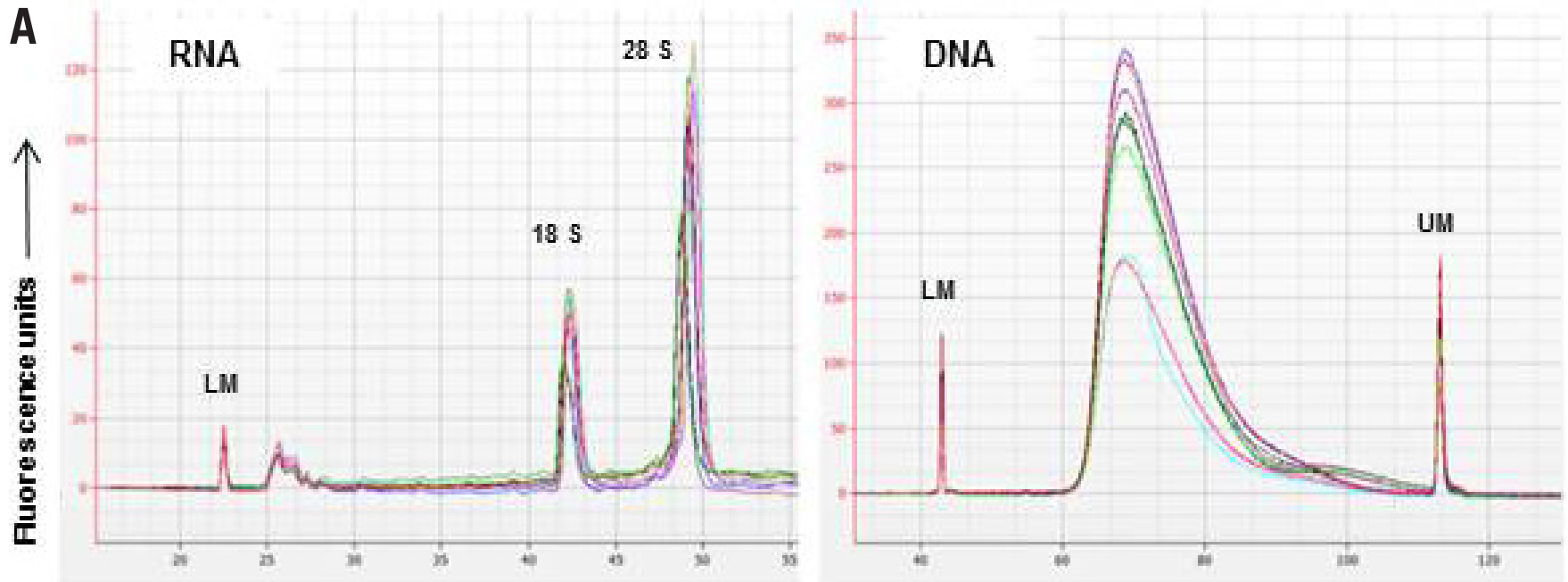

Elution time (seconds)

B

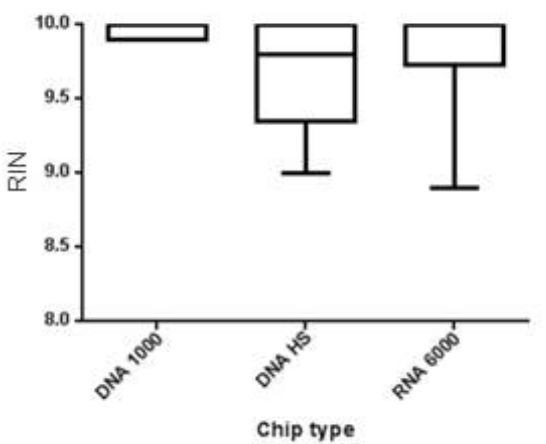

Bioanalyzer using the DNA HS assay. Manual integration was used to label the prominent peak for each RNA-Seq library, enabling library length and concentration to be calculated. The average length estimated across all Bioanalyzer chips was $290 \pm 6$ bp (mean \pm SD) (Table 2 and Figure 1), with a 40 bp range across all samples (inter-chip library length: $P=0.69$ by ANOVA). DNA concentration showed a 10\%-20\% variation across chips (Table 2). Accordingly, care should be taken when quanti-

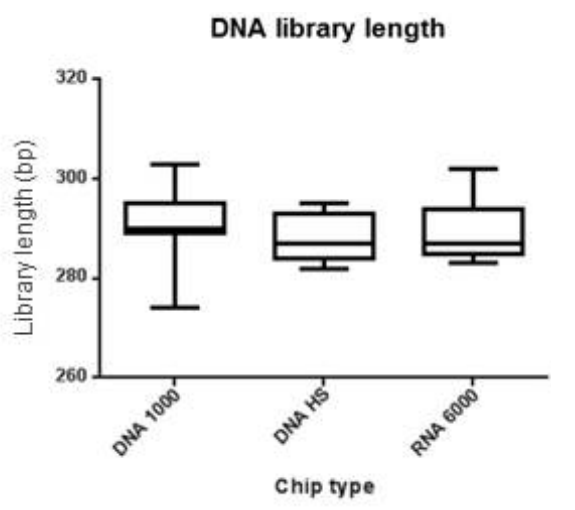

fying NGS libraries using the Agilent Bioanalyzer; however, the general recommendation is to use quantitative PCR (https://support.illumina.com/ downloads/sequencing_library_qper_ quantification_guide_11322363.html).

To assess assay reproducibility, which has been previously described by others (Agilent Application Note. Publication Number 5988-7650EN. 2002) (5), a pool of the RNA samples described above or a single DNA sample (RNA-Seq library pool) was loaded as technical replicates
Figure 1. Inter- and intra-chip consistency in the measurement of key sample parameters. (A) Overlaid electropherogram traces of all RNA and DNA Agilent Bioanalyzer runs. Four RNA or DNA samples were run in triplicate on RNA 6000 Nano, DNA High Sensitivity (HS), and DNA 1000 chips according to the RNA 6000 Nano or DNA HS kit protocols, respectively. Note that the lower fluorescence for the DNA 1000 chip did not affect calculation of sample concentration or library length. (B) RNA integrity number (RIN) for the four RNA samples and the library length of the four RNA-Seq libraries were determined on each chip. UM: upper marker peak; LM: lower marker peak.

in each well of duplicate chips for all three chip types tested. Library length and RIN were consistent between all chips (Figure 2). RNA and DNA concentration data were significantly different between chip types, although it was apparent that this was due to inter-chip variability and not chip type (Figure 2 and Supplementary Material).

We determined the accuracy of our Bioanalyzer concentration measurements by comparison to NanoDrop UV spectrophotometry (Nanodrop,

\begin{tabular}{|c|c|c|c|c|c|c|c|c|c|c|c|c|c|}
\hline & \multirow[t]{2}{*}{ RNA sample } & \multicolumn{4}{|c|}{ DNA 1000} & \multicolumn{4}{|c|}{ DNA HS } & \multicolumn{4}{|c|}{ RNA Nano } \\
\hline & & 1 & 2 & 3 & Mean & 1 & 2 & 3 & Mean & 1 & 2 & 3 & Mean \\
\hline \multirow[t]{3}{*}{ RIN } & R1 & 9.9 & 9.9 & 10.0 & 9.9 & 9.2 & 10.0 & 9.7 & 9.6 & 9.9 & 8.9 & 9.7 & 9.5 \\
\hline & R3 & 10.0 & 10.0 & 10.0 & 10.0 & 9.5 & 9.8 & 10.0 & 9.8 & 10.0 & 10.0 & 10.0 & 10.0 \\
\hline & R4 & 10.0 & 10.0 & 10.0 & 10.0 & n/a & $n / a$ & $\mathrm{n} / \mathrm{a}$ & $\mathrm{n} / \mathrm{a}$ & 10.0 & 10.0 & 10.0 & 10.0 \\
\hline \multirow{3}{*}{$\begin{array}{l}\text { Concentration } \\
\text { (ng/pl) }\end{array}$} & R1 & 147.0 & 147.0 & 138.0 & 144.0 & 151.0 & 131.0 & 129.0 & 137.0 & 151.0 & 161.0 & 122.0 & 144.7 \\
\hline & R3 & 323.0 & 318.0 & 337.0 & 326.0 & 343.0 & 280.0 & 299.0 & 307.3 & 254.0 & 272.0 & 355.0 & 293.7 \\
\hline & R4 & 222.0 & 231.0 & 265.0 & 239.3 & $n / a$ & $\mathrm{n} / \mathrm{a}$ & $\mathrm{n} / \mathrm{a}$ & $n / a$ & 227.0 & 238.0 & 261.0 & 242.0 \\
\hline
\end{tabular}

Four RNA samples were run according to the RNA 6000 Nano protocol on DNA 1000, DNA HS, and RNA 6000 Nano chips. RIN and sample concentration $(\mathrm{ng} / \mu \mathrm{l})$ were determined. Sample R4 was excluded from the DNA HS chip, as the final three wells were used to validate ladder consistency within a chip. 
A

RIN

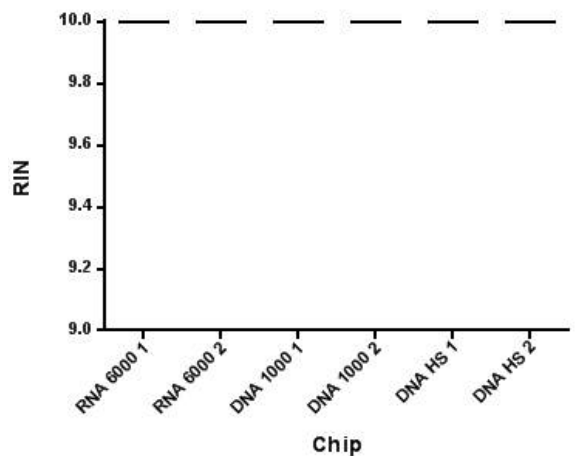

C

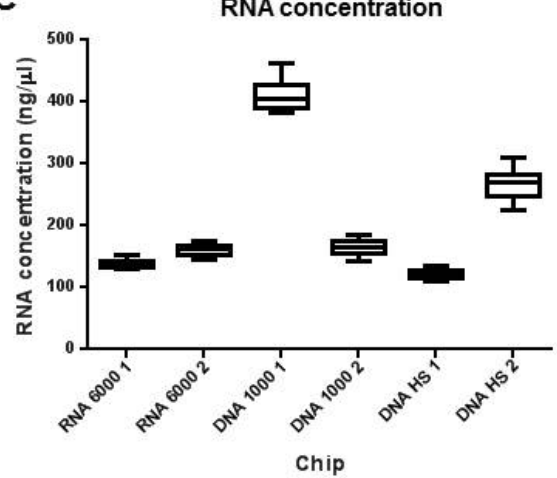

B

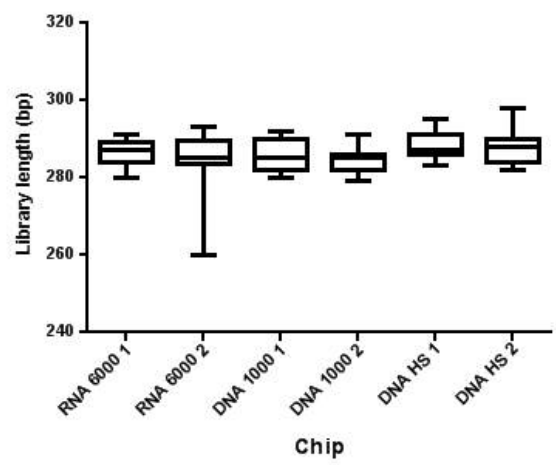

D

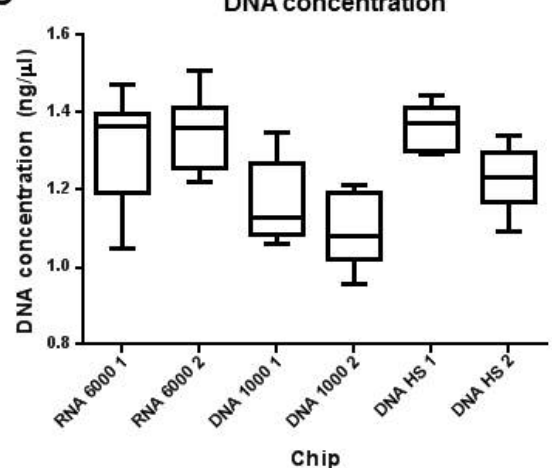

Figure 2. Between chip reproducibility. Technical replicates of one RNA or DNA sample were loaded onto duplicate chips to assess assay reproducibility. Both RNA and DNA quality (A and $B$ ) were highly reproducible. RNA and DNA concentration (C and D) showed high inter-chip variability. Tabulated data and results of statistical tests are given in the Supplementary Material.

Wilmington, DE), Qubit fluorometric quantitation (Thermo Fisher Scientific, Waltham, MA), and KAPA qPCR (KAPA BioSystems, Cape Town, South Africa). (Qubit and qPCR are the recommended quantification methods for RNA and RNA-Seq libraries, respectively.) The Bioanalyzer concentration measurement of the RNA pool was less precise $(211 \pm$ $102.89 \mathrm{ng} / \mathrm{\mu l}$ ) (mean $\pm \mathrm{SD}$ ) than Qubit (146 $\pm 6.24 \mathrm{ng} / \mathrm{\mu l})$. The Bioanalyzer underestimated the concentration of our RNA-Seq library $(1.25 \mathrm{ng} / \mathrm{\mu l})$ when compared with qPCR (11.17 ng/ $\mathrm{\mu l})$ (Supplementary Material). Our data support the finding that Bioanalyzer quantification is more variable than other methods (Agilent Application Note. Publication Number 5988-7650EN. 2002).

Our data confirm that, provided the assay-specific protocol is followed, the Bioanalyzer chip type used is irrelevant for RIN and DNA length estimation (i.e., qualitative analysis of RNA and RNA-Seq libraries). However, it is important that the chip sticker is not used as a guide. Qubit and GPCR are recommended for RNA and RNA-Seq library concentration measurements; the variability in RNA and DNA concentration measurements

\begin{tabular}{|c|c|c|c|c|c|c|c|c|c|c|c|c|c|}
\hline & \multirow[t]{2}{*}{ DNA sample } & \multicolumn{4}{|c|}{ DNA 1000} & \multicolumn{4}{|c|}{ DNA HS } & \multicolumn{4}{|c|}{ RNA Nano } \\
\hline & & 1 & 2 & 3 & Median & 1 & 2 & 3 & Median & 1 & 2 & 3 & Median \\
\hline \multirow{4}{*}{$\begin{array}{l}\text { Library length } \\
\text { (bp) }\end{array}$} & D1 & 274 & 284 & 291 & 284 & 287 & 285 & 282 & 285 & 286 & 285 & 283 & 285 \\
\hline & D2 & 303 & 294 & 297 & 297 & 294 & 292 & 295 & 294 & 295 & 294 & 290 & 294 \\
\hline & D3 & 290 & 290 & 290 & 290 & 282 & 285 & 284 & 284 & 286 & 285 & 287 & 286 \\
\hline & D4 & 295 & 289 & $\mathrm{n} / \mathrm{a}$ & 292 & 293 & 293 & $\mathrm{n} / \mathrm{a}$ & 293 & 302 & 293 & $\mathrm{n} / \mathrm{a}$ & 298 \\
\hline \multirow{4}{*}{$\begin{array}{l}\text { Concentration } \\
\text { (ng//ul) }\end{array}$} & D1 & 2.4 & 2.1 & 2.1 & 2.2 & 1.9 & 2.0 & 2.0 & 2.0 & 2.7 & 2.6 & 2.5 & 2.6 \\
\hline & D2 & 5.3 & 6.3 & 6.0 & 5.9 & 5.3 & 5.7 & 5.3 & 5.4 & 5.7 & 6.6 & 6.1 & 6.1 \\
\hline & D3 & 2.0 & 1.9 & 1.9 & 1.9 & 1.8 & 1.7 & 1.8 & 1.8 & 2.3 & 2.5 & 2.3 & 2.4 \\
\hline & D4 & 4.6 & 6.2 & $\mathrm{n} / \mathrm{a}$ & 5.4 & 4.7 & 5.3 & $\mathrm{n} / \mathrm{a}$ & 5.0 & 4.5 & 6.2 & $\mathrm{n} / \mathrm{a}$ & 5.3 \\
\hline
\end{tabular}

Four DNA samples (RNA-Seq libraries) were run in triplicate on each chip according to the DNA High Sensitivity (HS) protocol. Manual integration was used to delete artifacts and select the desired peak; library length (bp) and concentration (ng/ $\mu \mathrm{l}$ ) were determined by the software. The DNA HS protocol uses 11 sample wells, so Sample D4 was loaded in duplicate. we observed between Bioanalyzer runs supports this. These findings will be applicable to a number of research environments in which the Agilent 2100 Bioanalyzer is used for the assessment of nucleic acid samples, particularly those facilities that employ more than one type of kit and consume large quantities of Bioanalyzer reagents.

\section{Author contributions}

J.H. conceived the idea for the study. J.D. and T.D. performed the experiments. J.H., J.D., and T.D. analyzed data and wrote the manuscript.

\section{Acknowledgments}

We thank all members of the Genomics Core team past and present for useful discussions; Sarah Vowler for help with statistical tests; and Cancer Research UK and the University of Cambridge for funding the Genomics Core facilities through the Cambridge Institute grant.

\section{Competing interests}

The authors declare no competing interests.

\section{References}

1. Mueller, O., K. Hahnenberger, M. Dittmann, H. Yee, R. Dubrow, R. Nagle, and D. Ilsley. 2000. A microfluidic system for high-speed reproducible DNA sizing and quantitation. Electrophoresis 21:128-134.

2. Schroeder, A., O. Mueller, S. Stocker, R. Salowsky, M. Leiber, M. Gassmann, S. Lightfoot, W. Menzel, et al. 2006. The RIN: an RNA integrity number for assigning integrity values to RNA measurements. BMC Mol. Biol. 7:3.

3. Chim, W. and P.C.H. Li. 2012. Repeated capillary electrophoresis separations conducted on a commercial DNA chip. Anal. Methods 4:864-868.

4. Nguyen, T., S. Kwak, and S.J. Karpowicz. 2014. Re-use of commercial microfluidics chips for DNA, RNA, and protein electrophoresis. Biotechniques 57:267-271.

5. Lu, C.-Y., D.-J. Tso, T. Yang, Y.-J. Jong, and Y.-H. Wei. 2002. Detection of DNA mutations associated with mitochondrial diseases by Agilent 2100 bioanalyzer. Clin. Chim. Acta 318:97-105.

Received 25 September 2015; accepted 05 January 2016.

Address correspondence to James Hadfield, Genomics Core Facility, Cancer Research UK Cambridge Institute, Li Ka Shing Centre, University of Cambridge, Robinson Way, Cambridge, CB2 ORE, UK. E-mail: James.Hadfield@cruk.cam.ac.uk

To purchase reprints of this article, contact: biotechniques@fosterprinting.com 